Revue d'histoire de l'Amérique française

REVUE D.HISTOIRE DE L'AMÉRIQUE FRANÇAISE

\title{
La présence protestante en Nouvelle-France
}

\section{Marc-André Bédard}

Volume 31, numéro 3, décembre 1977

URI : https://id.erudit.org/iderudit/303632ar

DOI : https://doi.org/10.7202/303632ar

Aller au sommaire du numéro

Éditeur(s)

Institut d'histoire de l'Amérique française

ISSN

0035-2357 (imprimé)

1492-1383 (numérique)

Découvrir la revue

Citer cet article

Bédard, M.-A. (1977). La présence protestante en Nouvelle-France. Revue d'histoire de l'Amérique française, 31(3), 325-349.

https://doi.org/10.7202/303632ar d'utilisation que vous pouvez consulter en ligne.

https://apropos.erudit.org/fr/usagers/politique-dutilisation/ 


\title{
LA PRÉSENCE PROTESTANTE EN NOUVELLE-FRANCE
}

\author{
MARC-ANDRÉ BÉDARD \\ Bibliothèque de la Législature \\ Assemblée nationale du Québec
}

On a beaucoup étudié la population canadienne-française en fonction de ses origines géographiques ou sociales. Peu d'historiens, cependant, se sont intéressés à retracer l'appartenance religieuse de cette même population. Pourtant l'homogénéité de croyance n'est pas parfaite sous le Régime français. On dénombre même plus de 800 réformés venus dans la colonie durant cette période.

Il n'y a pas lieu d'insister ici sur les premières tentatives de colonisation, tentatives faites par des marchands protestants pour établir des comptoirs pelletiers soit en Acadie, à Tadoussac et même à Québec. Les expéditions de huguenots français comme Roberval, Chauvin, de Monts sont assez bien connues. Il en va de même pour la famille des de Caen. Les querelles qui eurent lieu dans ces différents postes entre catholiques et protestants sont restées célèbres ${ }^{1}$.

Mais cette situation allait cesser en 1627 par la fondation de la Compagnie des Cent-Associés. Désormais, non seulement les protestants n'ont plus le droit de diriger des entreprises commerciales dans la colonie, mais il leur est formellement interdit de s'établir en Nouvelle-France. On pourrait croire qu'à compter de cette date, il n'est plus question de protestants sur les bords du Saint-Laurent... pourtant tel n'est pas le cas! Il y a présence de protestants au pays jusqu'à la toute fin de la domination française.

Pour bien évaluer ce groupe, il faut identifier avec précision chaque individu et en déterminer la province ou le pays d'origine, la profession ou le statut, ainsi que la religion pratiquée. En effet,

Pour les premières tentatives de colonisation protestante, voir Marcel Trudel, Histoire de la Nouvelle-France, I: Les vaines tentatives 1524-1603 (Montréal, Fides, 1963), 307 p.; II : Le comptoir 1604-1627 (Montréal, Fides, 1966\$, 554 p.

RHAF, vol. $31, \mathrm{n}^{\circ} 3$ (décembre 1977) 
si les pressions des autorités civiles et religieuses pour les convertir au catholicisme s'adressent à tous indifféremment, le Français et l'étranger, le marchand et l'homme de métier, le soldat et le captif, ne réagissent pas nécessairement de la même façon. On les retrouve cependant dans la colonie jusqu'en 1759, même si l'absence d'une Église protestante organisée rend leur avenir religieux toujours incertain.

\section{PERMANENCE DE LA PRÉSENCE PROTESTANTE}

Que ce soit dans la correspondance religieuse ou dans les écrits civils, la fréquence des allusions à la présence des protestants au pays démontre que l'article II de la charte de la Compagnie des Cent-Associés ne fut pas appliqué dans toute sa rigueur.

Après le bref intermède des Kirke, dès 1635, les Relations des Jésuites mentionnent la présence de protestants à Québec ${ }^{2}$. Avec I'organisation croissante de la jeune Égilise canadienne, ia iutte contre la présence des réformés s'intensifie. Comme pour marquer son désir bien arrêté de mettre bon ordre à la situation, la première messe solennelle célébrée par Monseigneur de Laval à Québec en 1659 est accompagnée de l'abjuration d'un hérétique ${ }^{3}$.

En 1661, l'évêque écrit à Rome pour faire part de la promesse du roi de ne pas permettre aux «hérétiques» de s'établir à Québec ${ }^{4}$. Pourtant les années passent et la situation ne change pas. En 1670, le prélat revient à la charge. Il adresse un mémoire en France visant à interdire l'accès des nouveaux territoires aux protestants ${ }^{5}$. Quarante-trois ans après l'interdiction formelle de la venue des protestants en Nouvelle-France, ceux-ci continuent à s'y établir malgré les protestations du clergé.

L'Église, se rendant bientôt compte de l'inutilité de la lutte pour empêcher la venue de tous ces protestants, tentera alors de

2 Relations des Jésuites (Édition de Reuben Gold Thwaites), 8: 62; 11: 72.

3 Lettre de Mgr de Laval au pape Alexandre VII, 31 juillet 1659, Église du Canada, VII: 1 (Extraits des archives du Vatican concernant l'Église du Canada, conservées aux archives de l'archevêché de Québec).

4 Archives de la Propagande, 21 octobre $1661 \ldots$ cité dans Étienne-Michel Faillon, Histoire de la colonie française en Canada (Paris, Jacques Lecoffre et Cie, 1865), 170.

5 Mémoire de l'Évesque de Québec sur les Protestants, 1670, ASQ, Manuscrit 17, 207. 
limiter leur activité. En 1676, le Conseil Supérieur de Québec édicte de nouvelles politiques concernant les réformés. On y stipule que les protestants n'ont pas le droit de s'assembler pour célébrer le culte, mais on leur permet officiellement de séjourner au pays durant l'été. On va même jusqu'à permettre leur établissement permanent dans certains cas particuliers ${ }^{6}$.

Évidemment, l'évêque de Québec ne se tient pas pour battu et continue de faire pression pour limiter l'entrée au pays de ces réformés. En 1683, le ministre de la Marine assure l'évêque que les protestants ne viendront plus s'établir en Acadie ou à Québec ${ }^{7}$. Pourtant, la même année, des protestants français obtiennent la permission d'établir une pêche sédentaire en Acadie ${ }^{8}$.

Ainsi, à la veille de la révocation de l'Édit de Nantes en 1685, les protestants continuaient à être tolérés au pays. La révocation de l'Édit marque un ralentissement dans l'arrivée des dissidents religieux au Canada. Les protestants pourchassés en France ne tiennent pas à retrouver le même climat d'intolérance dans les colonies.

Ce n'est qu'après la mort de Louis XIV, en 1715, que les protestants recommencent à venir au Canada. En 1725, les plaintes du clergé reprennent ${ }^{9}$. Il en fut ainsi jusqu'à la fin de la domination française. En 1741, l'intendant Hocquart est obligé d'écrire en France pour rassurer le ministre de la Marine concernant la conduite des protestants au pays. Il répondait ainsi à une lettre de l'évêque qui s'était plaint du nombre croissant des protestants en Nouvelle-France ${ }^{10}$.

En 1749, le même scénario se répète et Bigot rassure les administrateurs de la métropole alertés encore une fois par le clergé ${ }^{11}$. En 1753, la situation reste inchangée, dit l'évêque et, quatre ans plus tard, le prélat constate laconiquement «que les choses restent

6 Extrait des «reglemens generaux du Conseil Superieur de Québec, pour la Police, du $11^{\mathrm{e}}$ mai $1676 »$, art. XXXVII.

7 Lettre du ministre à l'évêque, 3 août $1683, \mathrm{AC}$, Série $\mathrm{B}, 10$, fol. 8.

8 M. Dudouyt à Mgr de Laval, 26 mai 1682, ASQ, Lettres N, no 62: 5-7. fol. 47 .

9 Lettre de l'évêque de Québec au ministre, 19 octobre 1725, AC, Série C11A,

10 Lettre du président du Conseil de Marine à Beauharnois et Hocquart, le 20 avril 1741, AC, Serie B, 72 : fol. $3161 / 2$.

11 Lettre de Bigot au ministre, 3 octobre 1749, AC, Serie C11A, 93, fol. 257 et 258. 
toujours dans le même état» et que les protestants continuent de s'établir ${ }^{12}$.

Malgré les décrets explicites, malgré les pressions réitérées du clergé, il y eut donc, tout au long du Régime français, présence permanente des protestants dans la colonie. Cette présence éclatera au grand jour lors de l'entrée des troupes anglaises à Québec en 1759. Un soldat britannique pourra noter dans son journal que lors d'un service protestant célébré en actions de grâces pour la prise de la ville, plusieurs protestants français y assistaient avec les soldats anglais ${ }^{13}$.

\section{ORIGINE DES PROTESTANTS DE LA NOUVELLE-FRANCE}

Pour bien apprécier la diversité qui existe sous l'appellation générale de réformé, il est important de distinguer, pour chaque individu, le pays d'origine, la profession exercée et l'Église réformée dont il se réclame. Ces regroupements permettent de micux saisir l'importance relative des différentes catégories de protestants qui sont venus au Canada sous le Régime français.

La recension des réformés de la Nouvelle-France constituait un travail ardu. Il s'agissait de les retracer et de donner, dans la mesure $\mathrm{du}$ possible, tous les renseignements qui les concernent. Cette démarche qui n'avait jamais été effectuée auparavant a nécessité une foule de recherches. Elle nous a conduit à dénombrer, pour la durée du Régime français, un total de 853 personnes d'origine protestante ${ }^{14}$.

Il s'est avéré impossible, cependant, de retenir tous les noms de protestants rencontrés. Plusieurs d'entre eux ne sont mentionnés qu'une seule fois, au hasard d'un document, et il n'est plus jamais question d'eux par la suite. C'est le cas notamment de nombreux pri-

12 Lettre de l'évêque à L'Isle-Dieu, 30 octobre 1757, AC, Série C11A, 102. Les marchands protestants de la fin du Régime français ont été étudiés dans un article de J. F. Bosher paru en 1974 dans la Revue d'histoire sociale, VII, 14 (novembre 1974): 179-201.

13 Journal du capitaine Knox... cité dans Marcel Trudel, «Le protestantisme s'établit au Canada", La Revue de l'Université Laval, 10, 1 (septembre 1955): 3-22.

14 Pour plus de détails sur la recension des protestants de la colonie, voir Marc-André Bédard, "Les protestants en Nouvelle-France», thèse de maîtrise présentée à l'École des gradués de l'Université laval en 1973. 
sonniers anglais soignés à l'Hôtel-Dieu de Québec. Nous n'avons retenu que les 471 protestants sur lesquels nous avions des données suffisantes quant à leur origine et à leur situation dans la colonie.

\section{Lieu d'origine}

Les protestants se divisent en deux groupes: les Français et les étrangers. Si le contingent de la métropole française est le plus considérable, le nombre d'étrangers ne manque pas de surprendre (Tableau I).

\section{TABLEAU I}

\section{ORIGINE NATIONALE DES PROTESTANTS}

\section{Lieu d'origine}

France

Angleterre

Nouvelle-Angleterre

Allemagne

Suisse

Écosse

Irlande

Hollande

Flandres

Jamaïque

Nouvelle-Hollande

Suède

Inconnu

TOTAL

\section{Nombre de représentants}

192

152

75

47

21

19

12

10

4

1

1

1

1

Plusieurs étrangers ont séjourné et se sont même établis à demeure au pays sous la domination française. Les insinuations du Conseil Supérieur de Québec contiennent les «actes de naturalité » de plus de 130 personnes d'origine étrangère ${ }^{15}$. Cette liste d'ailleurs est loin de rendre compte de la totalité des protestants étrangers établis au Canada, puisque plusieurs l'ont fait sans recevoir de naturalisation française.

15 Pierre-Georges Roy, "Les lettres de naturalité sous le Régime français ", BRH, 30, 8 (août 1924): 225-232. 
Il est très difficile de retracer toutes ces personnes, car les notaires et les différents scribes de cette époque ont transcrit et fait évoluer constamment l'orthographe des noms étrangers. Sous leur plume Willet devient Ouellet et Formsworth se change en Fanef, Phanef et Phaneuf. Parfois un hasard permet de recouper deux documents, mais bien souvent des individus ne peuvent plus être identifiés à cause de ces multiples variations d'orthographe attribuables aux textes de l'époque.

La plupart de ces étrangers sont, soit des prisonniers anglais qui ont préféré s'établir au Canada, soit des soldats étrangers incorporés aux troupes métropolitaines envoyées dans la colonie. Il n'est donc pas étonnant d'y retrouver toutes les nations qui fournissent des soldats à la France, comme l'Allemagne et la Suisse. Mais les représentants les plus nombreux sont les ressortissants d'Angleterre et de Nouvelle-Angleterre amenés ici au cours des différents conflits coloniaux. Ils comptent respectivement 152 et 75 représentants.

Les protestants français (Tableau II) qui arrivent au Canada viennent de La Rochelle pour ie très grand nombre. Comme le fait remarquer Gabriel Debien, le rôle de La Rochelle dans la colonisation a été prépondérant au dix-septième siècle ${ }^{16}$. Or, comme cette ville du sud-ouest était le château fort du protestantisme, il n'est pas étonnant qu'elle fournisse à elle seule au moins 78 représentants des 192 protestants français retracés. Même après le siège de 1628, la ville de La Rochelle n'avait pas retrouvé d'un seul coup ses institutions catholiques. Dans un milieu qui, dès 1560 , s'était détaché massivement du catholicisme, toute l'organisation était demeurée majoritairement protestante. C'est donc de cette région que vint le plus fort contingent des réformés français.

En plus des protestants de La Rochelle et des environs, les régions du sud de la France ont aussi fourni une bonne part des réformés. Le Poitou, la Guyenne, la Saintonge, le Languedoc et le Périgord envoient 34 ressortissants. La Normandie, pourtant province du Nord, surprend avec ses 15 représentants. Il est vrai que ces personnes sont des marchands provenant de milieux urbains comme Rouen et ne sont pas représentatives, en ce sens, de la Normandie agricole et catholique. Des 155 Français dont l'origine provinciale

16 Gabriel Debien, «Les engagés pour le Canada au XVII ${ }^{\mathrm{e}}$ siècle vus de La Rochelle», RHAF, 6 (1952-1953): 205-206. 
est connue, la région du sud-ouest fournit à elle seule $72,3 \%$ de ces dissidents religieux ${ }^{17}$.

TABLEAU II

PROVENANCE DES PROTESTANTS FRANÇAIS

Lieu d'origine

Nombre de représentants

La Rochelle

78

Saintonge

16

Normandie

15

Poitou

Angoumois

7

Languedoc

Montauban

Paris

Périgord

Alsace-Lorraine

Bretagne

Guyenne

Bordeaux

Provence

Rochefort

Fontainebleau

Touraine

Vivarais

Inconnu

TOTAL

\section{Provenance sociale}

L'absence en France et dans la colonie d'un état civil protestant rend très difficile la tâche de reconstituer avec précision leur

17 Dans leurs analyses, généalogistes et démographes ont négligé la question de l'appartenance religieuse. Mgr Cyprien Tanguay, dans son Dictionnaire généalogique, semble avoir passé volontairement sous silence l'origine protestante de certaines personnes, car il ne pouvait ignorer l'existence du Registre d'Abjurations conservé à l'archevêché de Québec. Quant au père Archange Godbout, s'il a relevé minutieusement l'origine religieuse, il s'est plus attaché à la mobilité de la population («Familles venues de La Rochelle en Canada», RAPQ, 48 (1970): 113-377). Enfin, Hubert Charbonneau, dans un volume publié en 1975, retient l'occupation, mais non la religion des immigrants (Vie et mort de nos ancêtres. Étude démographique (Montréal, PUM, 1975), 267 p.). C'était là, croyons-nous, omettre un facteur de distinction profondément enraciné chez les individus. 
répartition sociale et professionnelle. Par provenance sociale, il faudra donc entendre ici une acception assez large. Il s'agit parfois de la profession exercée par un individu dans la colonie ou encore de la situation qu'il occupe lors de son arrivée (Tableau III). Pour considérer l'ensemble des protestants, nous avons distingué les prisonniers et les captifs. Il ne s'agit pas d'une profession, mais ils forment deux groupes particuliers qui ont été importants dans la colonie à une époque donnée.

TABLEAU III

\section{RÉPARTITION DES PROTESTANTS SELON LEUR STATUT*}

\begin{tabular}{lr}
\hline Soldats & 11 \\
Prisonniers & 76 \\
Captifs & 72 \\
Habitants & 62 \\
Marchands & 24 \\
Marins & 10 \\
Charpentiers-menuisiers & \\
Employés au chantier naval & \\
Officiers & 3 \\
Chirurgiens & \\
Forgerons & \\
Cordonniers & \\
Tailleurs & \\
Tapissier & \\
Secrétaire & \\
Boucher & \\
Laquais & \\
Meunier & \\
Bourreau & \\
Orfèvre & Écuyer \\
Journalier &
\end{tabular}

TOTAL 411

Il va sans dire que certains individus ont exercé plus d'une profession. Nous les avons alors considérés par rapport aux diver-

* Ce tableau indique le statut ou les professions exercées par les protestants venus durant le Régime français. Comme l'occupation de certains d'entre eux n'a pu être retracée avec précision et que certains autres en ont exercé plus d'une, ce tableau ne donne qu'une idée approximative. Il permet cependant d'avoir une image générale des protestants de la colonie. 
ses occupations exercées: il en va ainsi de plusieurs soldats qui, après s'être établis dans le pays, se sont intégrés à différents groupes sociaux.

\section{Les marchands}

Le commerce a toujours été une occupation privilégiée par les protestants. Ces marchands agissent parfois ou occasionnellement pour leur propre compte, mais la plupart du temps, ils sont les agents de grandes maisons françaises de La Rochelle d'abord, puis ensuite de Rouen et de Montauban. En 1741, quand l'évêque de Québec se plaint de la présence des marchands protestants, l'intendant et le gouverneur envoient en France une liste qui montre bien la fonction d'associé et de représentant des compagnies françaises remplie par plusieurs marchands réformés de Québec.

Le sieur Payé y est dit «associé de Raudy Père et fils de Montauban», Mounier est "commis du sieur Vessière de La Rochelle», les frères Thouron sont «associés et commis du sieur Boudet de La Rochelle», François Havy et Jean Lefèvre sont «associés et correspondants» de la compagnie Dugard de Rouen, et David Turpin est «commis» de la compagnie Le Vieux également de Rouen ${ }^{18}$.

Bien souvent ces marchands ne s'établissent pas de façon définitive. Ils font des affaires durant un certain nombre d'années, puis repassent en France. Le très petit nombre d'inventaires de biens de marchands protestants retracés en Nouvelle-France en est un bon indice. Les greffes des notaires contiennent les inventaires de cinq marchands réformés dont trois ont abjuré le protestantisme à Québec ${ }^{19}$.

D'autres marchands protestants se sont également établis de façon permanente, mais il a été impossible de retracer leur testament ou inventaire de biens. Pour toute la durée du Régime français, nous notons la présence d'au moins 25 marchands réformés. Ils sont d'origine française à l'exception de quatre étrangers. L'importance de leur titre commercial respectif va du simple commis négociant qui se rapproche du marchand général, au grand traiteur pelletier associé des maisons métropolitaines.

18 Lettre de Beauharnois et Hocquart au ministre, le 18 septembre 1741, AC, Série C11A, 75, fol. 14-15.

19 Pierre-Georges Roy, Inventaire des testaments, donations et inventaires du Régime français conservés aux Archives Judiciaires de Québec (Québec, 1941), 3 vol. 


\section{Les soldats}

Parmi les troupes françaises envoyées dans la colonie, il y eut toujours des protestants que les autorités religieuses locales s'efforcèrent de convertir. Dès 1665 , plus d'une vingtaine de soldats du régiment de Carignan-Salières ont déjà fait abjuration à l'Hôtel-Dieu de Québec ${ }^{20}$.

Certains auteurs protestants ont commis une erreur grave dans l'estimation du nombre de soldats protestants en Nouvelle-France. Ils ont confondu «réformé religieux» et "réformé militaire». Le pasteur Duclos, qui a écrit une Histoire du protestantisme français au Canada et aux États-Unis ${ }^{21}$, donne une liste de hauts personnages qui, selon lui, sont tous des calvinistes en $1690^{22}$. Pour établir cette liste, il se base sur les écrits de Charlevoix qui parle fréquemment de «capitaine réformé» et de «lieutenant réformé» ${ }^{23}$. Duclos conclut erronément qu'il s'agit de protestants alors qu'il ne s'agit en fait que de militaires à la retraite ou qui ont été mis à la demi-solde. Certains témoignages prouvent d'ailleurs qu'il s'agit bien de militaires et non de protestants. La Hontan rapporte une écheile de salaires des officiers dans la colonie. Il déclare que les soldes sont les suivantes: "Les capitaines des Troupes ont cent vingt livres par mois. Les Lieutenants quatre-vingt-dix livres, les Lieutenans Reformez cinquante, les Sous-Lieutenans quarante ${ }^{24}$ ». Certains auteurs anglais qui ont cité Duclos ont encore aggravé cette erreur d'interprétation en traduisant «réformé» par «calvinist». Ils affirment que ces personnes étaient calvinistes alors qu'il s'agit toujours de militaires réformés et non de protestants. Il était pourtant impossible que les hauts dignitaires de la colonie soient des protestants en 1690 , soit cinq ans après la révocation de l'Édit de Nantes qui excluait tous les protestants des charges publiques, ce qui inclut évidemment les postes militaires importants.

L'armée, cependant, compta toujours dans ses rangs des soldats qui appartenaient à la réforme. Les étrangers incorporés aux régi-

20 Dom Albert Jamet, Les Annales de l'Hôtel-Dieu de Québec 1636-17I6 (Montréal, Presses de Garden City, 1939), 148.

21 R.-P. Duclos, Histoire du protestantisme français au Canada et aux ÉtatsUnis (Montréal, Librairie Évangélique, s.d.,), 2 vol.

22 Ibid., 1: 30.

23 Charlevoix, Histoire et description générale de la Nouvelle-France (Paris, P.-F. Giffart, 1744), 2: 63, 86, 115, 117, 168, 290.

24 La Hontan, Voyages du baron de La Hontan dans l'Amérique septentrionale (Amsterdam, François L'Honoré, 1728), 2: 79. 
ments français arrivent surtout au dix-huitième siècle. Il s'agit dans la plupart des cas d'Allemands ou d'Anglais qui combattent pour le roi de France. Bien souvent les textes de l'époque les appellent des «soldats suisses", mais cette appellation ne fait pas toujours référence à une nationalité précise. Déjà en 1727 , le roi accorde la permission aux soldats suisses de s'établir dans la colonie à condition qu'ils se convertissent au catholicisme ${ }^{25}$. Plus tard, en 1747, arrive au Canada le régiment de Karrer composé exclusivement de suisses de nationalité allemande et anglaise. Plusieurs de ces soldats abjurèrent le protestantisme pour pouvoir s'établir au pays. Entre 1750 et 1759 , il y aura ainsi remontée spectaculaire des abjurations à Québec.

\section{Habitants et hommes de métiers}

Les grandes entreprises à caractère nettement spéculatif et les différentes compagnies de colonisation furent très influentes dans le recrutement des colons tout au long du dix-septième siècle ${ }^{26}$. Il n'entrait guère dans leurs préoccupations premières de filtrer les arrivées de protestants: d'ailleurs, à cette époque, la nécessité du peuplement de la colonie passait avant tout. Même une entreprise à caractère religieux comme celle des fondateurs de Ville-Marie recrutera des protestants pour Montréal en $1659^{27}$. À compter de l'hiver de 1659-1660, les protestants sont présents à Montréal et quelques-uns d'entre eux abjurent dès la première année de leur séjour ${ }^{28}$.

Dans l'apport de colons protestants, l'élément féminin est nettement inférieur en nombre à l'élément masculin. Les protestantes de la Nouvelle-France sont souvent de jeunes captives de la Nouvelle-Angleterre élevées dans la colonie; peu viennent de France.

25 Lettre du président du Conseil de Marine à $\mathbf{M}$. de St-Ovide et de Mésy, 10 juin 1727, AC, Série B, 50, fol. 579.

26 Debien, loc. cit., 185.

27 Gustave Lanctôt, "Position de la Nouvelle-France en 1663 ", RHAF, 11, no 4 (mars 1958): 522. M. Lanctôt confond la recrue de 1653 avec celle de 1659 cependant. D'après les témoignages de Marguerite Bourgeoys et de Dollier de Casson, c'est la recrue de 1659 qui comporte des éléments protestants. Voir Dollier de Casson, Histoire du Montréal (Montréal, La Minerve, 1868), 140. Voir également: Étienne-Michel Faillon, Vie de sæur Bourgeoys, fondatrice de la congrégation de Notre-Dame de Villemarie en Canada, suivie de l'histoire de cet institut jusqu'à ce jour (Ville-Marie), 1: 111-112.

28 Faillon, ibid., 2: 361. 
Cette proportion inférieure est d'ailleurs signalée par Debien qui remarque que les listes d'engagements de La Rochelle comportent «peu de ménages (et) peu de femmes isolées ${ }^{29}$ ». Le petit nombre de femmes protestantes s'inscrit dans le problème général de toute colonie à ses débuts: la faiblesse numérique de l'élément féminin. En Nouvelle-France, cette carence fut en partie comblée par la venue des «Filles du Roy». Comme ces jeunes filles sont souvent choisies dans des institutions caritatives catholiques, très peu d'entre elles appartiennent à la religion protestante. Dans un ouvrage paru en 1972, Silvio Dumas ne mentionne que deux filles du roi de religion protestante: Catherine Basset et Marthe Quitel ${ }^{30}$. Dans la liste qu'il donne en fin de volume, nous avons pourtant retracé 11 autres filles du roi qui sont de la religion réformée d'après la recension du père Archange Godbout ${ }^{31}$.

À cette liste de filles du roi recensées par Dumas (quoique non mentionnées comme d'origine protestante), il faudrait inclure également trois autres protestantes que l'auteur n'a pas recensées dans soñ ouiviage: Marguerite Doucinet, Isabclle Targer et Barbe Valade ${ }^{32}$. Le nombre total des filles du roi protestantes s'établirait donc à 16 représentantes.

\section{Les prisonniers}

Plusieurs soldats, anglais pour la plupart, mais aussi écossais et irlandais, faits prisonniers au cours des différents conflits qui opposaient la France et l'Angleterre en Amérique, furent ramenés à Québec. Les deux arrivées majeures de ces prisonniers se font à l'occasion de la prise du fort Saint-Jean de Terre-Neuve en 1709 et la prise du fort Chouagen durant la guerre de Sept Ans.

29 Debien, loc. cit., 216.

30 Silvio Dumas, Les filles du roi en Nouvelle-France (Québec, Société historique de Québec, 1972), 381 p.

31 Archange Godbout, loc. cit., 113-377. Il s'agit de: Catherine Barré, Madeleine De Launay, Isabelle ou Elisabeth Doucinet, Madeleine Dutault, Marie Léonard, Anne Lépine, Marie Mazoué, Barbe Ménard, Marguerite Navarre, Marie Targer et Marie Valade.

32 M. Dumas a sans doute confondu ces trois filles avec leur sœur qu'il avait déjà recensée. Marguerite Doucinet est la sœur d'Élisabeth, Isabelle Targer et Barbe Valade sont les sœurs de Marie. Il s'agit pourtant de personnes bien distinctes. Le père Archange Godbout signale le nom de leur époux respectif et la date de leurs différents mariages. Voir ibid., 213-214; $354 ; 363$. 
Comme les prisons de la ville n'étaient pas assez grandes pour recevoir toutes ces personnes, plusieurs d'entre elles furent distribuées dans les communautés religieuses et chez différents habitants de la ville. Ces prisonniers vivaient ainsi dans une demi-liberté au service de différentes maisons du pays. Ce contact avec les habitants favorisa sans aucun doute leur intégration et certains d'entre eux préférèrent rester au Canada lors des échanges de prisonniers. ${ }^{33}$

\section{Les captifs}

Les captifs diffèrent des prisonniers en ce sens qu'il ne s'agit pas de soldats de carrière, mais de civils de Nouvelle-Angleterre. Parmi eux se trouvent des hommes, des femmes et même des enfants en bas âge. Ils étaient ramenés dans la colonie à l'occasion de raids effectués par les Canadiens et leurs alliés indiens en Nouvelle-Angleterre. Certains d'entre eux, des enfants surtout, étaient directement rachetés par les missionnaires, aux Indiens qui les avaient enlevés à leurs parents.

Plusieurs de ces captifs ne retournèrent jamais dans leur pays lors des traités de paix. Le gouverneur de Boston écrit à Vaudreuil, en 1711, pour demander avec insistance que tous ces captifs puissent retourner en Nouvelle-Angleterre. Vaudreuil répond au gouverneur Dudley qu'il ne forcera pas à s'en retourner «ceux qui voudront de leur bon gré et volonté demeurer ${ }^{34}$ ».

À l'instar des prisonniers, ces captifs étaient placés dans différentes habitations de la ville ou dans des communautés religieuses. Les enfants en bas âge ne semblent pas avoir fait abjuration et étaient tout simplement baptisés et éduqués dans la foi catholique. Les plus âgés abjuraient pour un bon nombre ou se mettaient à pratiquer extérieurement le catholicisme. Vers 1730, les captifs du début du siècle ont souvent pris des surnoms français après avoir contracté mariage. D'autres ne sont nommés que par leur prénom français reçu au baptême ou par le nom de la famille où ils résident; «Marie» et «Joseph» étant des prénoms très répandus, il devient impossible de les distinguer.

33 Voir tableau, p. 332.

34 Lettre de Vaudreuil à M. Dudley, le 16 juin 1711, AC, Série C11A, fol. 94. 


\section{Appartenance religieuse}

Tous les protestants établis en Nouvelle-France n'appartiennent pas à la même Église réformée. Ils se répartissent en différentes dénominations souvent caractéristiques de leur pays d'origine.

Les protestants français sont désignés la plupart du temps par le sobriquet de «huguenots» ou encore sont appelés «membres de la religion prétendue réformée»: il s'agit en fait de calvinistes. Quand le terme calviniste est utilisé pour désigner des étrangers, il s'agit la plupart du temps d'Écossais.

Les Anglais sont membres de leur Église nationale dans une très grande proportion et s'appellent donc anglicans. Cependant, il y a parmi eux quelques dissidents religieux calvinistes. De leur côté, les individus venant de Nouvelle-Angleterre se dénomment anglicans, puritains, ou disent appartenir à une secte indépendante. Les luthériens de la colonie sont pour la plupart des soldats allemands venus dans les troupes de la métropole.

Ainsi il $y$ a prédominance de huguenots ou calvinistes parmi les Français, de luthériens chez les Allemands et d'anglicans chez les Anglais. Mais souvent les actes officiels ne donnent pas de façon précise la dénomination religieuse des réformés, se contentant de les désigner par le terme générique de protestant ou de prétendu réformé.

\section{LE SORT RÉSERVÉ AUX PROTESTANTS DE LA NOUVELLE-FRANCE}

Durant tout le Régime français, les protestants eurent à subir des pressions qui visaient à leur faire rejoindre les rangs de l'Église catholique. Ces mesures tenaient soit de la persuasion, soit de la coercition. Les non-catholiques tentèrent de réagir à la situation qui leur était faite, mais cette résistance ne prit jamais la forme d'une lutte ouverte et organisée.

\section{Les mesures prises contre les protestants}

\section{L'abjuration}

La renonciation au protestantisme se faisait par la signature d'un acte d'abjuration qui avait lieu à l'église paroissiale ou dans les chapelles des différentes communautés religieuses des villes. 
Ces abjurations étaient le fruit d'un travail incessant du clergé, des Jésuites surtout, qui voyaient à déceler les protestants et à les amener à poser le geste de l'abjuration. Durant les dernières années du règne de Louis XIV, l'autorité civile incita même le clergé à travailler en ce sens ${ }^{35}$. Durant les autres périodes, le clergé s'occupa seul de cette tâche en étant rarement secondé par les administrateurs de la colonie. Même si vers la fin du Régime français le président du Conseil de Marine invitait encore les autorités à travailler de concert avec l'évêque concernant la politique à suivre vis-à-vis les réformés, il n'était plus question cependant de pousser le clergé à faire abjurer les protestants ${ }^{36}$.

Les actes d'abjuration qui nous sont parvenus sont loin de rendre compte de la totalité des protestants convertis. Il existait des modèles-types qui étaient envoyés dans les paroisses et les dessertes et plusieurs de ces actes n'ont jamais été rapportés à Québec pour y être consignés ${ }^{37}$.

Toutes ces abjurations ne se faisaient pas par conviction personnelle. Il est évidemment impossible de distinguer si tel individu renonce au protestantisme par conviction ou à cause des pressions exercées sur lui. Il est cependant facile de voir que certaines époques comportent des conversions de groupe, comme en 1665 par exemple, où plus d'une vingtaine de personnes abjurent. Le catéchuménat était parfois réduit à sa plus simple expression et la signature de l'acte d'abjuration semble avoir été dans certains cas plus importante que la préparation spirituelle de l'individu à convertir.

Les méthodes utilisées pour obtenir les abjurations des protestants surprennent parfois par leur manque total de sérieux, voire de respect concernant la religion. Certains individus condamnés pour des délits mineurs se voyaient offrir des remises de peine s'ils acceptaient de se convertir ${ }^{38}$. D'autres étaient attirés par les pensions royales offertes aux protestants qui rejoignaient les rangs de l'Église. Denonville écrit en France en 1686 pour souligner que cette façon de faire utilisée dans la métropole «ferait bon effet». Il y aura

35 Mémoire de Louis XIV à MM. Denonville et de Champigny, 30 mars 1687, AC, Série B, 13, fol. 277.

${ }_{36}$ Lettre du président du Conseil de Marine à Vaudreuil, le 15 juillet 1755, AC, Série B, 101, fol. 138.

37 L'existence de ces modèles d'abjuration est mentionnée dans un inventaire fait au Séminaire de Québec. ASQ, Séminaire-92, 25: 62.

38 Jamet, op. cit., 148. 
même des demandes explicites de pension dans la colonie dans le cas de Hugues Cochrane dit Floridor et de Isaac Berthier ${ }^{39}$. En 1702, une somme de deux mille livres fut envoyée de France pour les Anglais qui se sont convertis au catholicisme au Canada ${ }^{40}$. Cette politique n'est pas sans rappeler l'existence des «caisses de conversions » qui étaient utilisées en France pour «stimuler» les abjurations des réformés ${ }^{4}$.

Pour toute la durée du Régime français, nous avons retracé un total de 256 abjurations ${ }^{42}$.

\section{Les actes de culte catholique}

Une autre façon de s'assurer de la pratique religieuse des protestants était de les contraindre à poser des actes de culte catholique, surtout à l'occasion de trois moments importants de la vie: la naissance d'un enfant, le mariage et la sépulture.

Une ordonnance édictée en France obligeait les parents protestants à présenter leur enfant à l'église catholique pour y être baptisé dès le jour de sa naissance. La même méthode fut utilisée au Canada et non seulement les enfants captifs de Nouvelle-Angleterre étaient baptisés à l'église catholique, mais aussi les enfants des protestants français. Monseigneur de Laval fit proclamer une ordonnance en 1664 enjoignant de faire baptiser les enfants à l'église dès leur naissance. Il revient sur le même sujet en 1677 constatant qu'elle n'était pas suivie avec rigueur. Il spécifie alors que tous les parents «de quelque qualité et condition qu'ils soient» doivent faire baptiser leurs enfants aussitôt après leur naissance ${ }^{43}$. Cette ordonnance de l'évêque ne visait pas uniquement les protestants, mais c'était une façon efficace d'obliger ces derniers à présenter leurs enfants à l'église pour les faire baptiser. Cette façon de faire conduisait même parfois les parents à

39 Denonville au ministre, 1686, AC, Série C11A, 8; Lettre de Talon au roi, 7 octobre 1665, AC, Série C11A, 2.

40 Mémoire du roi à Denonville, 3 mai 1702, AC, Série B, 23, fol. 41.

41 Émile G. Léonard, Histoire générale du protestantisme (Paris, PUF, 19611964), 2: 357-358.

42 Quelques actes d'abjuration sont sans doute rapportés dans les registres de diverses paroisses fondées sous le Régime français. L'inventaire de tous ces registres paroissiaux dépassait évidemment le cadre de ce travail.

43 Ordonnance du 5 février $1677 \ldots$ cité dans Auguste Gosselin, Vie de Mgr de Laval, premier évêque de Québec et apôtre du Canada, 1662-1708 (Québec, Imprimerie L.-J. Demers et Frères, 1890), 2: 43. 
demander l'abjuration avant de faire baptiser leur enfant. En 1756, la veuve Anne Nosget abjure à Notre-Dame de Québec quelques jours avant de faire baptiser son enfant nouveau-né ${ }^{44}$.

Les protestants étaient également obligés de se marier à l'église catholique. Ils n'avaient pas le choix d'ailleurs, puisqu'il n'y avait ni pasteur, ni lieu de culte réformé. Ainsi tous les protestants qui se sont mariés dans le pays l'ont fait devant un prêtre catholique. Quand il était reconnu que l'un des conjoints était de religion protestante, il devait faire abjuration de son hérésie à l'occasion de son mariage. C'est ainsi que 11 protestants abjurent quelques jours avant la célébration.

En ce qui concerne les funérailles, une ordonnance française spécifiait que les protestants devaient accepter de recevoir les derniers sacrements sous peine de voir leurs biens confisqués s'ils recouvraient la santé. Nous n'avons pas retrouvé d'application de cette dernière ordonnance en Nouvelle-France. Nous avons constaté cependant que des protestants ont attendu d'être à l'extrême limite de leur vie pour poser le geste de l'abjuration. Est-ce qu'ils craignaient l'application de l'ordonnance dont il est question plus haut ou s'agissait-il de conversion sincère? Un autre motif de crainte a pu jouer également dans la conversion tardive de ces individus. En effet, les protestants qui mouraient sans avoir renoncé à leur croyance étaient enterrés dans les champs. Il n'était pas question pour eux d'avoir une sépulture en terre bénite. Les archives de l'HôpitalGénéral de Québec mentionnent parfois les noms de ces réformés qui «voulant mourir dans la religion protestante (furent inhumés) dans un champ des environs de l'hôpital ${ }^{45}$ ».

En plus de ces trois actes de culte, l'administration du sacrement de confirmation à des personnes adultes permettait aussi de déceler les protestants et de les obliger à poser un acte de culte catholique. Refuser de se faire confirmer était par le fait même se dénoncer et s'exposer à des représailles. Le fait que le premier registre des confirmations conservé aux archives de l'archevêché de Québec se trouve sur les mêmes feuilles que le premier registre des abjurations constitue peut-être un indice du rapprochement fait entre l'abjuration et la confirmation dans la foi catholique des adultes de la

44 AAQ, Registre des Abjurations, no 163.

45 AHG, Registre des Minuttes sérié 1728-1783, 56, 84, 93, 100, 102, 104. 
colonie $^{46}$. La confirmation des protestants adultes était peut-être une forme plus douce d'abjuration réservée à des personnes qui s'étaient comportées extérieurement comme des catholiques depuis un certain nombre d'années. Ce vieux «Cathalogue» des confirmations contient le nom de certains protestants qui n'ont jamais abjuré et ont quand même reçu le sacrement des mains de l'évêque de Québec ${ }^{47}$.

\section{Les dragonnades}

Les méthodes énumérées jusqu'à maintenant pour forcer les protestants à passer au catholicisme ne font nullement mention de violence physique à leur endroit. De telles méthodes furent pourtant utilisées en France et sont désignées généralement sous le nom de dragonnades. Il s'agissait d'obliger les familles protestantes à loger dans leur maison des soldats spécialement désignés à cette fin.

Dans son Histoire du protestantisme français au Canada et aux États-Unis, le pasteur Duclos affirme, sans donner de références, que des violences physiques furent exercées sur les protestants de la colonie et même que certains furent emprisonnés et tués ${ }^{48}$. Il est vrai que des lettres furent envoyées au Canada pour dragonner les protestants. Dans une lettre parvenue à Denonville en 1686, il est fait mention des nombreuses conversions obtenues en France depuis la révocation de l'Édit de Nantes. La lettre continue en disant que, si parmi les protestants du pays il s'en trouve qui refusent de s'instruire, il a la permission «de se servir des soldats pour mettre garnison chez eulx, ou les faire mettre en prison, en joignant à cette rigueur le soing nécessaire pour les instruire, en quoy il doit agir de concert avec l'Evesque ${ }^{49}$ ». Ainsi il est exact que la latitude de dragonner fut donnée, mais rien toutefois n'indique que ces mesures aient

46 Mgr de Laval accordait beaucoup d'importance à ce sacrement. Lors de son arrivée au Canada en 1659, le premier geste officiel qu'il pose en arrivant à Percé est de confirmer 130 personnes à cet endroit, des marins de La Rochelle pour un bon nombre. Voir: Lettre de Mgr de Laval à Alexandre VII, le 31 juillet 1659, AAQ, Église du Canada, 7: 1.

${ }^{47} \mathrm{AAQ}$, Cathalogue ou Registre des Confirmés. En pied se trouve le Registre tes Abjurations.

48 Duclos, op. cit., 1: 22.

49 Mémoire du Roy à Denonville, 31 mai 1686, AC, Série B, 12: fol. 25 sq. 
effectivement été appliquées ${ }^{50}$. Quoiqu'il en soit, nous sommes loin des nombreux protestants emprisonnés ou tués sous le Régime français. Aucun texte ne l'atteste.

\section{Autres mesures utilisées contre les protestants}

Les autorités civiles disposaient enfin d'une série de mesures indirectes pour forcer les protestants à adhérer au catholicisme.

Parmi ces dernières, il y avait l'exclusion explicite de certaines occupations et professions. Dans les archives du Séminaire de Québec se trouve un texte manuscrit résumant les professions interdites aux protestants ${ }^{51}$. Le fait que ces défenses soient ainsi colligées et résumées sur une feuille spéciale indique sans doute que ces ordonnances, édictées en France entre 1680 et 1685, furent envoyées dans la colonie pour y être appliquées. Les métiers de médecin, apothicaire et sage-femme sont nommément défendus aux protestants. Il en va de même pour les fonctions de contrôleur, commis, brigadier, archer et huissier. Ils ne peuvent non plus être greffier, procureur, notaire, juge, ni remplir aucune fonction dans la maison royale. Ce texte indique enfin qu'un protestant ne peut être nommé tuteur et qu'un enfant mineur protestant peut se marier sans la permission de ses parents, à condition de recevoir le consentement de parents, amis ou voisins catholiques ${ }^{52}$.

Il est facile de comprendre que l'exclusion de certaines professions devait faciliter la conversion rapide de quelques réformés. L'occupation de négociant restait, il est vrai, toujours ouverte aux protestants, mais là encore des tracasseries de toute sorte venues des autorités les guettaient. En 1667, le négociant Daniel Bialle fut arrêté pour avoir fait décharger des marchandises à Montréal, un jour de fête, au mépris de la religion catholique ${ }^{53}$. Gabriel Bernon se vit refuser le droit d'hiverner au Canada à cause de ses convictions religieuses. Denonville écrit au ministre en 1685 pour dire qu'il aimerait bien le garder dans la colonie, mais que l'évêque s'y oppose ${ }^{54}$.

so Le cas de l'exécution du protestant Daniel Vuil (ou Vial) pour le commerce de l'eau-de-vie n'est pas, à proprement parler, un cas de persécution religieuse. G. Lanctôt l'a bien démontré dans un article paru dans SCHEC (1944-45): 11-25, «Une accusation contre Mgr de Laval».

51 ASQ, Polygraphie-IV, no 4.

52 Ibid.

53 Jugements du Conseil Souverain de la Nouvelle-France (Québec, A. Côté, 1885), $1: 442$.

54 Lettre de Denonville au ministre, 13 novembre 1685 , AC, Série C11A, 11. 
Finalement, ce marchand repassa en France et, de là, il partit avec des coreligionnaires pour aller fonder un établissement près de Boston 55 .

En ce qui concerne les protestants d'origine étrangère qui désiraient devenir sujets français, les lettres de naturalité spécifiaient que seuls les catholiques pouvaient en recevoir ${ }^{56}$. Ainsi, dans les lettres de naturalité insinuées en Nouvelle-France, il est toujours mentionné que les récipiendaires font profession de foi catholique ${ }^{57}$. C'était une autre façon efficace de pousser les protestants à abandonner leur religion.

Ces pressions s'exerçaient même au sein de la famille. Outre l'obligation de faire baptiser les enfants à l'église, les parents devaient en confier l'éducation aux institutions catholiques, sous peine d'y être contraints. Ainsi en 1689, la fille d'un nommé Pasteur est envoyée au couvent des Ursulines. Le ministre de la Marine approuve ce geste et ajoute:

Le roi laisse la liberté d'obliger les nouveaux convertis dont la conduite n'est pas assez exacte à y envoyer leurs filles pour leur apprendre les devoirs de la religion et y estre gardez jusqu'à ce qu'on trouve à les marier à de bons catholiques ${ }^{58}$.

\section{Réaction des protestants}

Les protestants de la colonie réagirent rarement comme groupe à ces mesures. Leurs réactions sont à caractère individuel et vont de l'opportunisme à la résistance passive. Il y eut très peu de cas de résistance active.

Plusieurs des protestants établis au Canada se comportent extérieurement comme de bons catholiques et réussissent à être considérés comme tels par les autorités religieuses. Ils vont à la messe et observent tous les préceptes de l'Église d'une façon générale. C'est ainsi que bon nombre de ces individus ne furent jamais considérés

55 Benjamin Sulte, «Gabriel Bernon», BRH, 22, no 1 (janvier 1916): 19-21.

56 Le ministre à MM. de Vaudreuil et Raudot, le 10 mai 1710, AC, Série B, 32, fol. $7 \frac{1}{2}$.

57 Pierre-Georges Roy, Inventaire des Insinuations du Conseil Souverain de la Nouvelle-France (Beauceville, L'Éclaireur, 1921), 121 et 130.

s8 Lettre du ministre au sieur Parat, le 7 juin $1689 \ldots$ cité dans Collection de manuscrits contenant lettres, mémoires, et autres documents relatifs à la NouvelleFrance (Québec, Imprimerie A. Côté et Cie, 1883), 1: 454. 
comme des protestants, puisque leur conduite extérieure ne permettait pas de déceler leur allégeance religieuse. Cette façon de faire était assez répandue puisque le nombre des protestants qui n'ont jamais abjuré, tout en n'étant pas inquiétés par les autorités religieuses, demeure très élevé. Dans le volume du père Godbout sur les engagés originaires de la ville de La Rochelle ${ }^{59}$, très rares sont les protestants qui furent par la suite obligés d'abjurer publiquement leur religion. Ils se soumettaient donc aux mesures prises contre eux en faisant baptiser leurs enfants à l'église et en remplissant toutes les obligations des catholiques.

Parfois ces personnes étaient quand même reconnues par les autorités religieuses et, même après s'être comportées longtemps comme catholiques, devaient poser le geste de l'abjuration. En 1646, le Journal des Jésuites rapporte le fait suivant: «un nommé Thomas (...) natif de La Rochelle qui jusques alors n'avait esté qu'apparemment Catholique et au fond estait Huguenot fit abjuration et profession de la foy ${ }^{60}$ ». D'autres individus qui s'étaient mariés à l'église et ne s'étaient pas déclarés protestants à cette occasion furent obligés d'abjurer par la suite.

Certains protestants vont aussi abjurer par opportunisme. Ainsi la coïncidence du mariage et de l'abjuration indique assez bien le motif poursuivi lors de cette abjuration: il s'agissait beaucoup plus du désir de se marier que de conversion intérieure. D'autres vont abjurer immédiatement avant de mourir. Encore ici, raison d'opportunisme: le désir de ne pas être enterré hors du cimetière et aussi, ne pas prendre le risque de se faire confisquer ses biens pour avoir refusé les derniers sacrements. Enfin le désir d'avoir des terres a pu jouer. Comme il était spécifié qu'aucun protestant ne pouvait s'établir dans la colonie, aucun protestant reconnu comme tel ne pouvait recevoir de concessions. Certaines abjurations furent ainsi motivées par le désir de s'établir. Il faut remarquer cependant que plusieurs protestants dont l'appartenance religieuse n'était pas connue réussirent à avoir des terres sans abjurer.

Il est difficile de savoir avec précision s'il y eut, comme en France, une organisation de l'«Église du Désert» dans la colonie. Cette appellation désigne tout le réseau de lieux de cultes et de ministres protestants inconnus des autorités qui, en France, circu-

59 Archange Godbout, loc. cit., 113-377.

60 Relations des Jésuites, 48: 190. 
laient parmi les habitations protestantes pour y lire la Bible et y célébrer le culte ${ }^{61}$. Comme toute cette activité se déroulait secrètement, il ne nous est parvenu aucun témoignage direct sur la pratique secrète du protestantisme en Nouvelle-France. Certaines remarques des autorités religieuses nous incitent à croire cependant qu'il y eut bel et bien une certaine organisation clandestine des protestants, même s'il est difficile d'en apprécier l'importance.

En 1670, Monseigneur de Laval écrit que les protestants «tiennent plusieurs discours seduisans, qu'ils prêtent des livres et que quelquefois ils se sont assemblés entre eux ${ }^{62}$. Si certaines assemblées ont été tenues et ont été connues de l'évêque, il est fort probable que plusieurs autres se déroulèrent sans jamais être découvertes. En 1749, Bigot réfute à son tour une accusation similaire portée par Monseigneur de Pontbriand ${ }^{63}$. S'il est impossible de retracer le nom d'un pasteur ou d'un lieu de culte, la tenue occasionnelle d'assemblée reste néanmoins plausible.

Enfin certains protestants se marièrent entre eux en NouvelleFrance. Ces mariages eurent lieu dans les églises cathoiliques, mais ie fait que certains protestants aient tenu à épouser un conjoint de la même religion que la leur est assez révélateur. La pratique extérieure catholique ne signifiait pas nécessairement l'abandon de ses croyances intérieures. Les nouveaux foyers continuaient, sans doute secrètement, à éduquer leurs enfants dans les mêmes principes religieux. C'est ainsi que le fils du huguenot Isaac Bédard, Jacques, épouse Isabelle Doucinet, également protestante, à Québec le 4 octobre $1666^{64}$.

Un dernier type de réaction protestante, plus radical celui-là, consiste à quitter la colonie et à fuir en Nouvelle-Angleterre pour $\mathrm{y}$ rejoindre des coreligionnaires. Les colonies anglaises servaient de refuge aux dissidents religieux européens. Plusieurs protestants français s'y étaient déjà établis en venant directement de France. Entre 1683 et 1687 , il est parfois question de la désertion de colons de la Nouvelle-France qui vont s'établir en Nouvelle-Angleterre. Denon-

61 Jean Delumeau, Naissance et affirmation de la Réforme (Paris, PUF, 1965) (Coll. «Nouvelle-Clio»), 245.

${ }^{62}$ Mémoire de l'Evesque de Québec sur les Protestants, 1670, ASQ, Manuscrit 17, 207.

63 Lettre de Bigot au ministre, le 3 octobre 1749, AC, Série C11A, 93, fol. 258.

64 Cyprien Tanguay, Dictionnaire généalogique (Québec, Eusèbe Sénécal, 1871), $1: 36$. 
ville écrit au ministre pour s'en plaindre. En 1687, on cite le cas de Gédéon Petit qui a «déserté aux Anglais ${ }^{65}$ ». Gédéon Petit était le fils du marchand protestant Alexandre Petit. Il avait abjuré le protestantisme à Québec le 20 mars $1673^{66}$. Lors de l'expédition envisagée sous Frontenac pour aller s'emparer de certains postes de la NouvelleAngleterre, il est spécifié que les protestants français établis en ces lieux devront être renvoyés en France ${ }^{67}$.

L'historien américain Charles W. Baird, qui s'appuie sur des documents sérieux, parle d'individus et de familles entières qui réussirent à s'enfuir de Nouvelle-France pour passer dans les colonies anglaises ${ }^{68}$. Il rapporte que plusieurs autres protestants désiraient poser le même geste, mais qu'ils en étaient dissuadés par la crainte des Indiens Maquas. C'est ce qu'affirmait un agent anglais envoyé à Québec lors d'un échange de prisonniers et qui racontait y avoir rencontré ces protestants français désirant s'enfuir du pays. Baird indique même que le gouverneur de la Nouvelle-France écrivit dans la métropole en 1683 pour dire qu'il y avait au-delà de soixante Français qui avaient déserté à Orange, «Manatte» et d'autres places hollandaises sous commandement anglais ${ }^{69}$. Il est improbable que toutes ces désertions aient été le cas de protestants qui désiraient quitter la colonie. Certaines personnes ont pu gagner les établissements anglais pour des raisons commerciales, comme la traite des fourrures. L'existence de certaines désertions pour des motifs religieux est tout de même établie par ces documents.

L'absence d'une Église organisée et d'institutions qui leur étaient propres greva lourdement les possibilités d'action des protestants de Nouvelle-France. Il n'existe aucun exemple où les protestants se soient associés pour protester en tant que groupe à la situation qui leur était faite. Même les marchands réformés qui consti-

65 Lettre de Denonville au ministre, le 8 juin 1687, AC, Série C11 A, 9.

66 AAQ, Registre des Abjurations, no 52.

67 Instruction à M. de Frontenac sur l'entreprise contre les Anglais, 7 juin 1689, ...cité dans Collection de manuscrits contenant lettres, mémoires, et autres documents relatifs à la Nouvelle-France (Québec, Imprimerie A. Côté et Cie, 1883), $1: 460$.

68 Charles W. Baird, History of the Huguenot Emigration to America (Baltimore, Regional Publishing Company, 1966), 1: 123.

69 Ibid. Baird affirme ces faits à partir des documents suivants:

1. Documents relative to the Colonial History of the State of New-York, 9: 203.

2. The information of Mathew Corey received from severall ffrench Protestants officiers and soldiers at Quebeck (oct. 28, 1695), Mass. Archives, A. 38. 
tuent une force économique avec laquelle les autorités doivent compter ne protestent pas comme groupe. Ils ne font aucune représentation auprès du Conseil Souverain pour améliorer leur condition d'hivernement ou d'établissement. Peut-être jugeaient-ils inutile d'entreprendre un combat qu'ils considéraient comme perdu d'avance.

À en juger par la réaction des protestants à la situation qui leur était faite, force nous est de constater que les pressions furent efficaces. Les cas de résistance passive existent et sont assez répandus, mais les réformés ne surent jamais faire entendre leur voix de façon collective. La réaction fut faible, individuelle et désorganisée.

\section{CONCLUSION}

Aux premières tentatives protestantes de colonisation avait succédé en 1627 une situation où les protestants étaient théoriquement exclus de la colonie. Malgré cette interdiction, des réformés français et étrangers continueront à venir tout au long de la domination française. Cette permanence de leur présence ne doit pas nous faire oublier cependant la précarité constante de leur situation. Totalement désorganisés, les protestants font face à une série de mesures visant à leur faire rejoindre les rangs de l'Église catholique. Dans ce contexte, l'esprit de tolérance était fréquemment absent et une telle situation laissait peu de place aux dissidents religieux qui désiraient vivre selon les exigences de leur conscience. En ce sens, les mesures prises contre les réformés furent efficaces. En effet, même s'il y a permanence de la présence des réformés, il est difficile d'y déceler le fruit d'une longue continuité qui se serait prolongée secrètement. Il s'agit plutôt du renouvellement constant des effectifs par de nouveaux arrivés; les protestants de la fin de la période étudiée ne sont pas les descendants de ceux arrivés un siècle plus tôt. Ceux-ci s'étaient convertis depuis fort longtemps. L'intolérance des autorités avait amené l'adhésion plus ou moins forcée des parents et, petit à petit, les enfants éduqués et noyés dans un milieu complètement catholique, étaient devenus eux-mêmes des catholiques. D'ailleurs, comment des individus privés d'institutions ecclésiales pour les soutenir auraient-ils pu continuer à maintenir une pratique vivace durant plusieurs générations?

Sous l'Ancien régime, cette intolérance n'était pas la marque exclusive des milieux catholiques. L'arrivée, en 1759, des nouveaux 
maîtres à Québec donna lieu aux mêmes manifestations d'esprit sectaire. Par le serment du Test, les catholiques vont goûter, pendant quelques années, au régime qu'ils avaient imposé durant si longtemps aux protestants. Tragique retournement de situation pour l'ancienne colonie française et catholique dont les destinées allaient maintenant être entre les mains de maîtres anglais et protestants. Un long apprentissage à la tolérance religieuse venait de commencer. 